\title{
Evaluación de la Anatomía Mandibular Relacionada con la Osteotomía Sagital de la Rama
}

\author{
Evaluation of Mandibular Anatomy Related to Sagittal Split Ramus Osteotomy \\ *Guillermo Salgado A; "Oscar Inzunza H; **,***Mario Cantín; ** Ramón Fuentes; \\ "Verónica Inostroza; "María Jesús Errázuriz \& *Cindy Pavez
}

SALGADO, A.G.; INZUNZA, H. O.; CANTÍN, M.; FUENTES, R.; INOSTROZA, V.; ERRÁZURIZ, M. J. \& PAVEZ, C. Evaluación de la anatomía mandibular relacionada con la osteotomía sagital de la rama. Int. J. Morphol., 30(1):30-39, 2012.

RESUMEN: La osteotomía sagital de la rama mandibular (SSRO) es una de las técnicas quirúrgicas más frecuentes para corregir las deformidades de la mandíbula. Con el fin de prevenir problemas anatómicos y quirúrgicos, los cirujanos requieren una mayor investigación sobre las estructuras anatómicas relacionadas con la SSRO. El objetivo de este estudio fue investigar las posiciones de la antilingula (AL), la entrada al nervio alveolar inferior (NAI) en la mandíbula y otros puntos de referencia anatómicos en relación con la língula mandibular (L). Fueron estudiadas 70 hemimandíbulas secas. La AL y los demás puntos de referencia y, la posición correspondiente de la L se marcaron en la cara medial y lateral de la rama mandibular respectivamente. Fueron medidas las distancias de la AL, NAI y L en los planos anterior-posterior y superior-inferior con un caliper, y se estableció su relación geométrica. Los resultados mostraron que la AL era perceptible en el 100\% de las caras laterales mandibulares. La mayoría de las ALs se encuentran anterior a la L, con una distancia media de $0,66 \pm 2,43 \mathrm{~mm}$ y $0,92 \pm 2,56 \mathrm{~mm}$ y, 4,23 $\pm 2,97 \mathrm{~mm}$ y 3,62 $\pm 3,14 \mathrm{~mm}$ superior a ella (lados derecho-izquierdo respectivamente) (ambos con un valor de $\mathrm{p}<0,001$ ). Valores similares se observaron en relación con el NAI. No se encontraron diferencias significativas entre los lados derecho e izquierdo, para la mayoría de los parámetros. Los parámetros estudiados pueden asistir a los cirujanos maxilofaciales a determinar la proximidad anatómica del NAI, y reducir al mínimo el riesgo de dañar el nervio y vasos sanguíneos. No recomendamos el uso de la AL como única referencia anatómica cuando se realiza un procedimiento de SSRO.

PALABRAS CLAVE: Osteotomía sagital de la rama; Mandíbula, Puntos de referencia anatómicos; Antilingula; língula; Nervio alveolar inferior.

\section{INTRODUCCIÓN}

La osteotomía sagital de la rama mandibular (SSRO) comprende una parte integral del tratamiento combinado de ortodoncia y cirugía de las deformidades dentofaciales mandibulares como el prognatismo y retrognatia, causadas por un sobre o bajo crecimiento de la mandíbula. Esta técnica (SSRO) fue popularizada por Trauner \& Obwegeser (1957) mediante el reporte de procedimientos de avances y retrocesos mandibulares. Las modificaciones de Dal Pont (Dal Pont, 1961; Epker, 1977) y Hunsuck (1968) se utilizan frecuentemente, transformándolo en un procedimiento de rutina en cirugía ortognática.

La SSRO tiene numerosas ventajas, como un abordaje intraoral, interfaz gama de hueso medular, cicatrización rápida y fácil, y permite la fijación rígida junto a una función mandibular temprana (Yu \& Wong, 2008). Sin embargo, no siempre existe un control visual de las estructuras anatómi- cas al realizar el procedimiento, resultando en daños postoperatoria (Plooij et al., 2009). Se han reportado múltiples complicaciones asociadas a la SSRO (O'Ryan, 1990; Precius et al., 1998; Turvey, 1985), principalmente la lesión del nervio alveolar inferior (NAI) y trastornos neurosensoriales en la región mentoniana (frecuencia de $2 \%$ $15 \%$ ) (Yu \& Wong). Otras corresponden a la fractura de los segmentos óseos distal o proximal antes o después de la cirugía (O’Ryan; Precius et al.; Turvey; Van Sockles et al., 1985; Plooij et al.), especialmente cuando se realiza la osteotomía horizontal medial durante la SSRO, junto a lesión de estructuras vasculares y fracturas no favorables en casos de corticales delgadas y ángulos amplios (Kim et al., 1997), sobre todo cuando la osteotomía horizontal medial se realiza en o por encima de un punto de fusión entre las corticales lateral y medial de la rama mandibular (Guernsey \& DeChamplain, 1971; Muto et al., 2003; Kim et al.; Ribeiro et al., 2006).

\footnotetext{
* Departamento de Anatomía Normal, Facultad de Medicina, Pontificia Universidad Católica de Chile, Santiago, Chile.

** Departamento de Odontología Integral, Facultad de Medicina, Universidad de La Frontera, Temuco, Chile.

**** Doctorado en Ciencia Morfológicas, Universidad de la Frotera, Chile.
} 
Conocer las características anatómicas de la rama mandibular y el curso de sus estructuras, así como sus variaciones, puede reducir el riesgo de lesiones durante la osteotomía (Fujimura et al., 2006). Se han realizado diversos estudios sobre las relaciones anatómicas existentes mediante técnicas radiográficas convencionales (Klinge et al., 1989) o topográficas (Hayward et al., 1977), las cuales tienen grandes limitaciones como la ampliación, distorsión y reproductibilidad cuestionable (Torres et al., 1991). En muchas ocasiones los cirujanos no son capaces de acceder de forma segura al sitio de la osteotomía utilizando como puntos de referencia los datos anatómicos sobre la posición del NAI y las relaciones geométricas entre otros puntos de referencia quirúrgica como la língula mandibular (L). Otro punto anatómico quirúrgico considerado como guía por los cirujanos es la posición del segundo molar inferior, sin embargo es inscontante según el caso, incluso en pacientes jóvenes (Yu \& Wong).

Diversos autores han establecido el uso de antilíngula (AL) como una guía para la realización de osteotomía horizontal medial, punto anatómico que ha sido discutido con diversas conclusiones en su relación con la L y el NAI (Rajchel et al., 1986; Yates et al., 1976; Hogan \& Ellis, 2006). Yi \& Wong evaluaron la anatomía mandibular relacionada con la osteotomía sagital en población taiwanesa mediante TC 3D, concluyendo que la posición anatómica del nervio y las relaciones geométricas entre la AL, L y el NAI a nivel del foramen mandibular muestran que la utilización de AL como guía quirúrgica es fiable. Esto fue recientemente confirmado por Apinhasmit et al. (2011).

Algunos estudios sobre mandíbulas humanas óseas

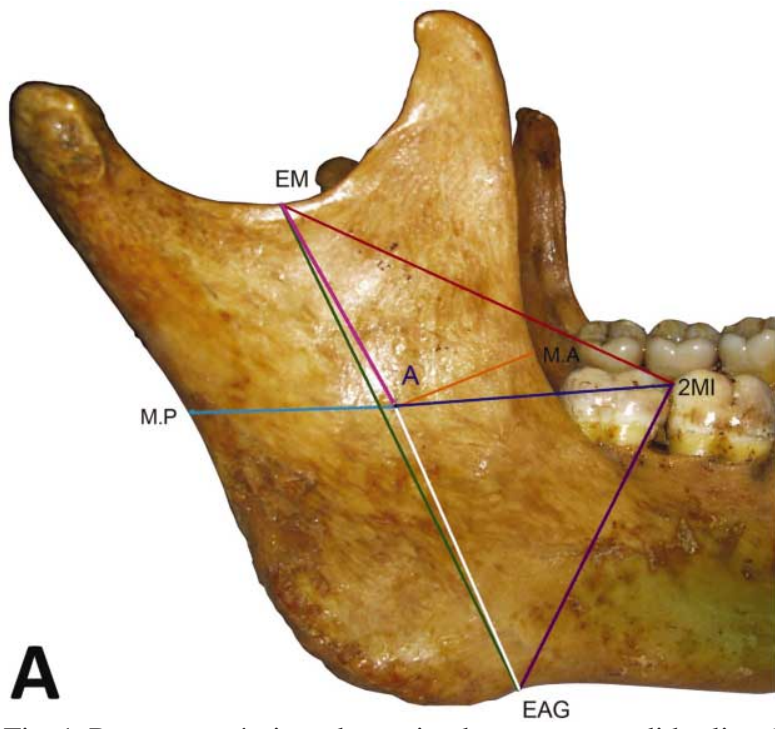

Fig. 1. Puntos anatómicos determinados con sus medidas lineales y angulares sobre la cara lateral (A) y medial (B) de la rama mandibular. EM: Escotadura Mandibular, EAG: Escotadura Antegonial, L: Língula, A: Antilíngula, NAI: Nervio Alveolar Inferior, 2MI: Segundo molar inferior, MA: margen anterior de la rama mandibular, MP: margen posterior de la rama mandibular.

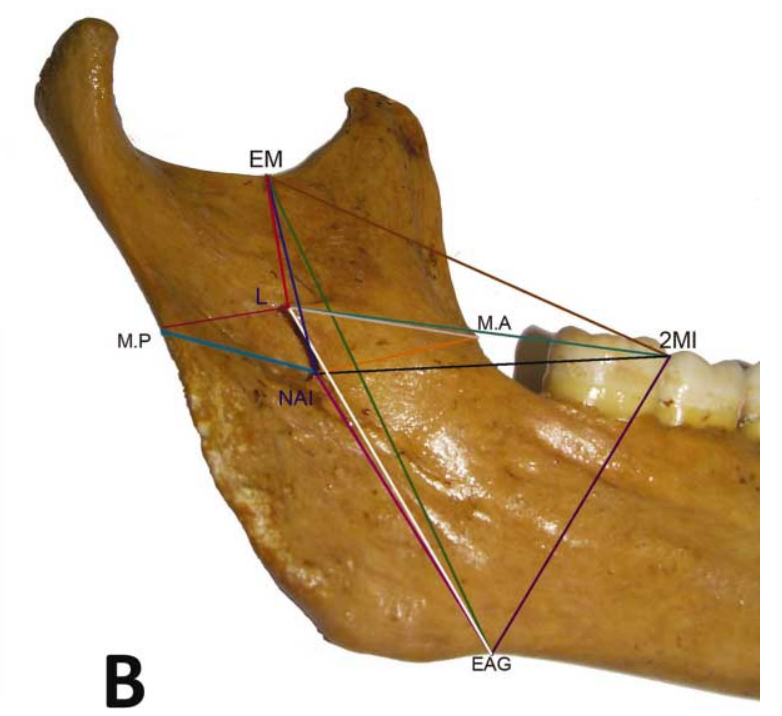

en poblaciones específicas han abordado la anatomía implicada en la osteotomía sagital (Fridrich et al., 1995; Kim et al.; Martone et al., 1993; Fujimura et al., 2006). Sin embargo, no existen estudios anatómicos directos que hayan relacionado la medición de las estructuras anatómicas clásicas implicadas en la SSRO y su relación geométrica con la AL. El objetivo de esta investigación fue determinar mediante el estudio directo sobre mandíbulas humanas secas las relaciones geométricas entre AL, L y el NAI en su entrada al canal mandibular, así como las relaciones con las estructuras que la rodean y que son de importancia para la realización de la SSRO.

\section{MATERIAL Y MÉTODO}

Se utilizaron 70 hemimandíbulas (35 mandíbulas) humanas adultas secas pertenecientes a población chilena del Departamento de Anatomía de la Facultad de Medicina, Pontificia Universidad Católica de Chile, las que presentan dentición total o parcial con el grupo molar intacto, sobre las cuales se realizaron mediciones lineales a través de un caliper. No se realizaron distinciones según sexo y edad.

Las mediciones se realizaron en la cara lateral y medial de las ramas mandibulares derecha e izquierda considerando como puntos anatómicos la escotadura mandibular (EM), escotadura antegonial (EAG), língula (L), Antilíngula (AL), entrada al canal mandibular del nervio alveolar inferior (NAI), segundo molar inferior (2MI), margen anterior 
de la rama mandibular (MA), magen posterior de la rama mandibular (MP). Luego, sobre las caras laterales mandibulares se proyectaron las siguientes distancias: EMAL, EM-EAG, AL-EAG, MA-AL, MP-AL, AL-2MI, EM2MI, EAG-2MI, y sobre las caras mediales mandibulares se registraron las siguientes: EM-NAI, EAG-NAI, EM-EAG, EM-L, EAG-L, MA-NAI, MP- NAI, MA-L, BP-L, EM-2MI, L-2MI, NAI-2 MI, EAG-2MI (Fig. 1 A y B).

Se analizaron valores longitudinales de la rama mandibular para establecer alguna relación existente entre

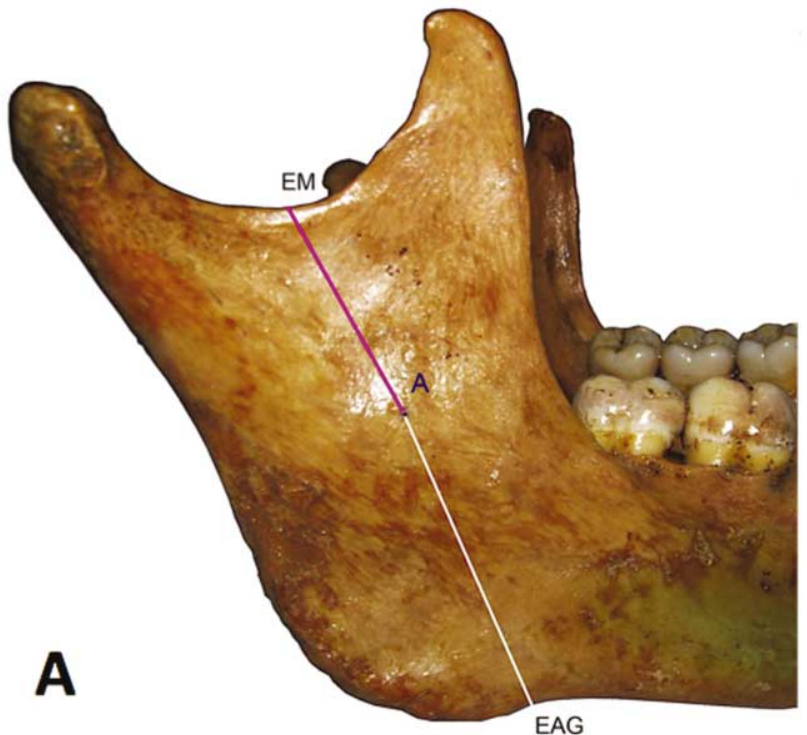

la AL como punto guía, con el NAI y la L. Tanto para la rama mandibular derecha como izquierda se comparó el promedio de la diferencia entre estas dos medidas las cuales se tomaron desde los siguientes parámetros:

Parámetro superior: usando como referencia la EM hacia la AL y L, se calculó la diferencia entre estos dos valores la cual correspondería a la distancia entre AL y L. Del mismo modo, se midió desde la EM hacia la AL y NAI calculándose la diferencia que habían entre estos dos valores la cual correspondería a la distancia entre AL y NAI.

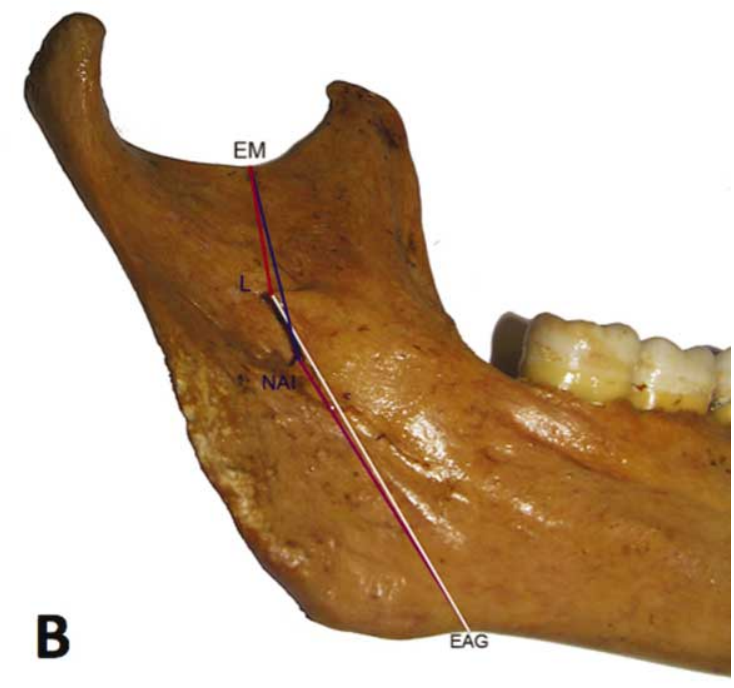

Fig. 2. Detalle de los puntos anatómicos utilizados para analizar los valores verticales sobre la cara lateral (A) y medial (B) de la rama mandibular divididos en parámetros superiores e inferiores. EM: Escotadura Mandibular, EAG: Escotadura Antegonial, L: Língula, AL: Antilíngula, NAI: Nervio Alveolar Inferior, 2MI.
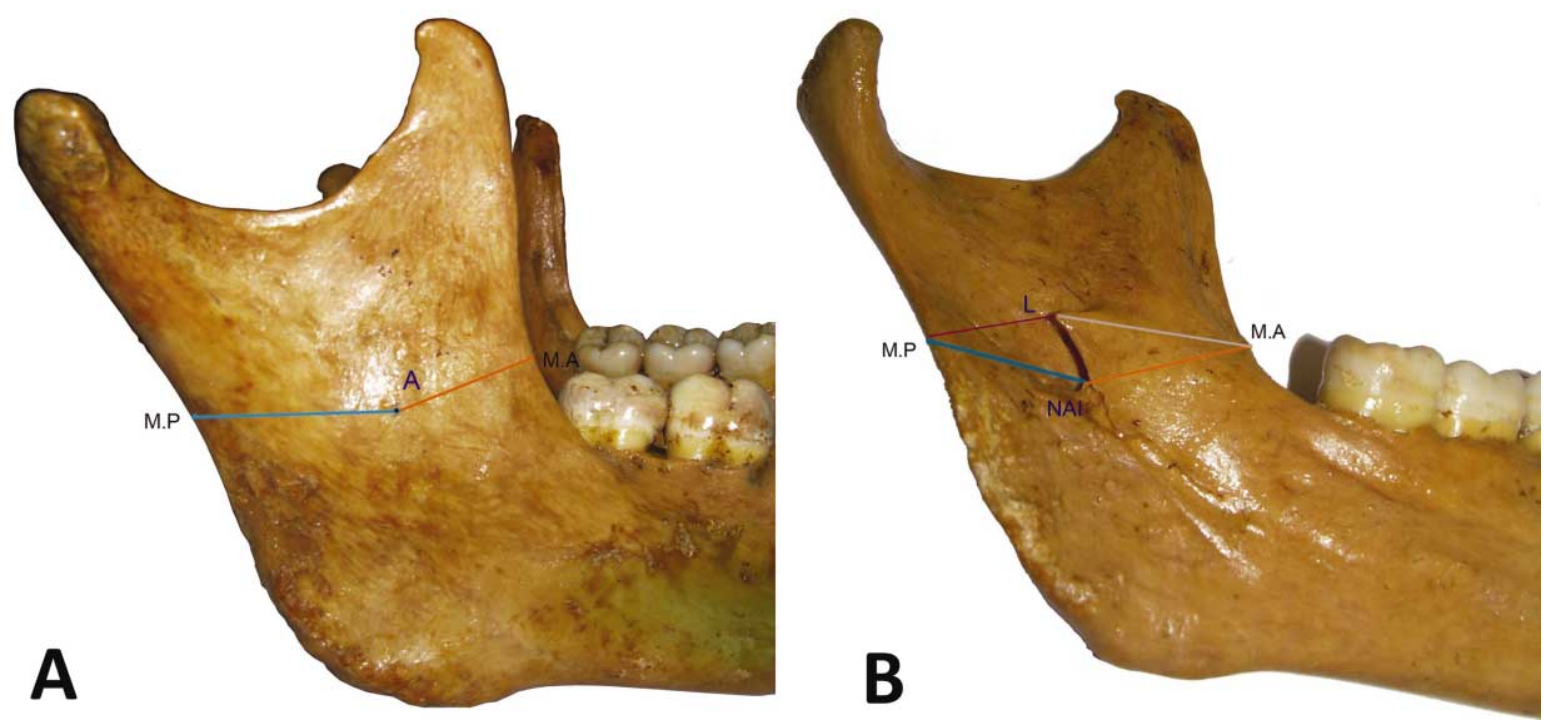

Fig. 3. Detalle de los puntos anatómicos utilizados para analizar los valores horizontales sobre la cara lateral (A) y medial (B) de la rama mandibular divididos en parámetros anteriores y posteriores. L: Língula, A: Antilíngula, NAI: Nervio Alveolar Inferior, 2MI: Segundo molar inferior, MA: Margen Anterior de la rama mandibular, MP: Margen Posterior de la rama mandibular. 
Parámetro inferior: usando como referencia la EAG y se analizó la diferencia, obteniendo la distancia entre los mismos puntos anteriores (AL, L y NAI) (Fig. 2 A y B).

Del mismo modo se analizó la relación que mantiene la AL en los valores horizontales (antero- posteriores) de la rama mandibular derecha e izquierda con los puntos anatómicos anteriormente mencionados (NAI y L). Los parámetros medidos fueron los siguientes:

Parámetro anterior: usando como referencia el MA hacia la AL y L, se calculó la diferencia entre estos dos valores la cual correspondería a la distancia entre AL y L. Luego, se siguió el mismo método para calcular la diferencia entre el MA hacia la AL y NAI calculándose la diferencia que habían entre estos dos valores la cual correspondería a la distancia entre AL y NAI.

Parámetro posterior: siguió el mismo método usando como referencia el MP y se analizó la diferencia obteniendo la distancia entre los mismos puntos anteriores (AL, L y NAI) (Fig. 3 A y B).

Una vez tabulados los datos de los valores obtenidos entre la cara lateral derecha e izquierda, y entre la cara medial derecha e izquierda de cada rama mandibular se realizó el análisis estadístico descriptivo e inferencial, con valores de $\mathrm{p}<0,01$ y $\mathrm{p}<0,001$.

\section{RESULTADOS}

Al comparar las mediciones entre los puntos anatómicos de la cara medial y la lateral con sus respectivas homólogas de las ramas mandibulares derecha e izquierda no se encontraron diferencias estadísticamente significativas observables entre ellas $(0,255>p>0,961)$ (Tablas I y II).

Tabla I. Distancia entre los puntos anatómicos de la cara lateral derecha e izquierda de las mandíbulas.

\begin{tabular}{lccccccc}
\hline & \multicolumn{3}{c}{ Cara lateral derecha } & \multicolumn{5}{c}{ Cara lateral izquierda } \\
\cline { 2 - 6 } & Promedio \pm DE & Máximo & Mínimo & Media \pm DE & Máximo & Mínimo & Valor p \\
\hline EM-AL & $13,22 \pm 2,48$ & 19,91 & 8,85 & $13,48 \pm 2,63$ & 20,01 & 8,80 & 0,668 \\
EM-EAG & $49,43 \pm 4,87$ & 60,94 & 39,35 & $48,60 \pm 5,68$ & 57,75 & 32,90 & 0,514 \\
AL-EAG & $36,78 \pm 4,57$ & 49,86 & 27,04 & $35,86 \pm 4,73$ & 43,30 & 24,46 & 0,411 \\
AL-MA & $15,35 \pm 2,37$ & 20,49 & 9,85 & $15,28 \pm 2,14$ & 19,83 & 10,70 & 0,902 \\
AL-MP & $15,48 \pm 1,97$ & 19,42 & 11,05 & $15,59 \pm 1,61$ & 20,14 & 12,99 & 0,793 \\
AL-2MI & $39,97 \pm 4,54$ & 43,25 & 24,67 & $34,45 \pm 4,23$ & 39,48 & 21,55 & 0,789 \\
EM-2MI & $41,14 \pm 4,84$ & 48,99 & 29,55 & $41,25 \pm 4,90$ & 49,21 & 28,85 & 0,838 \\
EAG-2MI & $33,07 \pm 3,56$ & 39,8 & 25,51 & $32,70 \pm 3,40$ & 38,78 & 25,57 & 0,675 \\
\hline
\end{tabular}

Tabla II. Distancia entre los puntos anatómicos de la cara medial derecha e izquierda de las mandíbulas.

\begin{tabular}{lccccccc}
\hline & \multicolumn{2}{c}{ Cara medial derecha } & \multicolumn{3}{c}{ Cara medial izquierda } \\
\cline { 2 - 6 } & Promedio \pm DE(mm) & Máximo & Mínimo & Media \pm DE $(\mathbf{m m})$ & Máximo & Mínimo & Valor p \\
\hline EM-NAI & $23,17 \pm 3,04$ & 32,86 & 14,60 & $22,78 \pm 2,45$ & 27,53 & 15,95 & 0,549 \\
EAG-NAI & $26,75 \pm 4,72$ & 35,49 & 11,97 & $27,01 \pm 4,10$ & 36,34 & 16,92 & 0,874 \\
EM-EAG & $49,15 \pm 4,79$ & 58,21 & 38,05 & $48,95 \pm 4,83$ & 56,71 & 33,73 & 0,860 \\
EM-L & $17,45 \pm 2,34$ & 24,84 & 12,56 & $17,10 \pm 2,05$ & 21,58 & 10,7 & 0,507 \\
EAG-L & $34,01 \pm 3,96$ & 40,59 & 22,76 & $34,09 \pm 4,53$ & 43,28 & 20,56 & 0,934 \\
NAI-MA & $16,16 \pm 1,69$ & 18,81 & 11,70 & $16,41 \pm 2,47$ & 20,17 & 6,54 & 0,288 \\
NAI-MP & $13,99 \pm 1,40$ & 16,86 & 10,15 & $13,59 \pm 1,47$ & 17,72 & 10,49 & 0,255 \\
L-MA & $16,01 \pm 2,51$ & 20,90 & 7,95 & $16,19 \pm 2,21$ & 20,54 & 10,5 & 0,744 \\
L-MP & $16,26 \pm 1,60$ & 20,19 & 12,78 & $15,46 \pm 1,77$ & 19,80 & 11,5 & 0,053 \\
EM-2MI & $41,24 \pm 4,64$ & 47,05 & 30,16 & $41,45 \pm 4,76$ & 49,18 & 30,08 & 0,838 \\
L-2MI & $30,90 \pm 4,73$ & 38,16 & 20,19 & $31,04 \pm 4,33$ & 36,93 & 20,23 & 0,961 \\
NAI-2MI & $30,16 \pm 4,21$ & 37,21 & 20,30 & $30,21 \pm 4,11$ & 35,82 & 19,98 & 0,927 \\
EAG-2MI & $32,41 \pm 4,13$ & 39,45 & 20,55 & $33,39 \pm 4,34$ & 48,71 & 24,61 & 0,366 \\
\hline
\end{tabular}


En la rama mandibular derecha los valores longitudinales de las distancias entre AL-L y AL-NAI fueron significativas para los parámetros de medida superior e inferior. Con el parámetro superior la distancia promedio entre AL-L fue de $4,23 \pm 2,97 \mathrm{~mm}$ ( $p<0,001)$ y entre AL-NAI de $9,95 \pm 3,16 \mathrm{~mm}(\mathrm{p}<0,001)$; con el parámetro inferior la distancia entre AL-L fue de $2,77 \pm 3,32(\mathrm{p}<0,009)$ y entre ALNAI de $10,03 \pm 3,86 \mathrm{~mm}(\mathrm{p}<0,001)$. En la rama izquierda, las distancias entre AL-L y AL-NAI fueron significativas para los parámetros de medida superior $(\mathrm{p}<0,001)$, siendo estas de $3,62 \pm 3,14 \mathrm{~mm}$ para AL-L y $9,3 \pm 3,39 \mathrm{~mm}$ para AL-NAI; en cambio con el parámetro de medida inferior la distancia significativa se encontró sólo entre AL-NAI con un valor de $8,84 \pm 4,14 \mathrm{~mm}(\mathrm{p}<0,001)$ en contraste con la distancia entre AL-L de sólo 1,76 $\pm 3,72 \mathrm{~mm}(\mathrm{p}=0,116)$ (Tabla III).

$\mathrm{Al}$ determinar los valores horizontales, en la rama derecha como en la rama izquierda la distancia entre ALNAI fue significativa desde el parámetro posterior con un valor de $1,49 \pm 2,12 \mathrm{~mm}(\mathrm{p}<0,001)$ y $2,00 \pm 1,94 \mathrm{~mm}(\mathrm{p}<0,001)$ respectivamente. Para el parámetro anterior, sólo se encontraron diferencias significativas en la rama izquierda para las distancias entre AL-NAI, la cual fue $1,13 \pm 2,6 \mathrm{~mm}$ ( $p=0,008)$, en contraste con la rama derecha, donde este valor fue de $0,82 \pm 2,24(\mathrm{p}=0,13)$.

No se encontraron distancias significativas para las distancias entre AL-L para ninguna rama mandibular ni para algún parámetro. En la rama derecha las distancias entre AL-L con los parámetros de medida anterior y posterior fueron de $0,66 \pm 2,43 \mathrm{~mm}(\mathrm{p}=0,26)$ y $0,78 \pm 2,24 \mathrm{~mm}(\mathrm{p}=0,073)$ respectivamente; del mismo modo para la rama izquierda fue de $0,92 \pm 2,56 \mathrm{~mm}(\mathrm{p}=0,083)$ y $0,13 \pm 2,08 \mathrm{~mm}(\mathrm{p}=0,755)$ (Tabla IV).

Tabla III. Diferencias de las distancias entre los valores longitudinales verticales de las mandíbulas.

\begin{tabular}{llcccc}
\hline & & \multicolumn{2}{c}{ Rama derecha } & \multicolumn{2}{c}{ Rama izquierda } \\
\cline { 3 - 6 } & & Promedio \pm DE $(\mathbf{m m})$ & Valor $\mathbf{p}$ & Promedio \pm DE $(\mathbf{m m})$ & Valor $\mathbf{p}$ \\
\cline { 2 - 5 } Parámetro & AL $-\mathrm{L}$ & $4,23 \pm 2,97$ & $<0,001 * *$ & $3,62 \pm 3,14$ & $<0,001^{* *}$ \\
Superior & AL-NAI & $9,95 \pm 3,16$ & $<0,001^{* *}$ & $9,30 \pm 3,39$ & $<0,001^{* *}$ \\
Parámetro & AL-L & $2,77 \pm 3,32$ & $0,009^{*}$ & $1,76 \pm 3,72$ & 0,116 \\
Inferior & AL-NAI & $10,03 \pm 3,86$ & $<0,001^{* *}$ & $8,84 \pm 4,14$ & $<0,001^{* *}$ \\
\hline
\end{tabular}

* Valor $\mathrm{p}<0,01 ; * *$ Valor $\mathrm{p}<0,001$

Tabla IV. Diferencias de las distancias entre los valores horizontales de las mandíbulas.

\begin{tabular}{llcccc}
\hline & & \multicolumn{2}{c}{ Rama derecha } & \multicolumn{2}{c}{ Rama izquierda } \\
\cline { 3 - 6 } & & Prome dio \pm DE $(\mathbf{m m})$ & Valor $\mathbf{p}$ & Promedio \pm DE $(\mathbf{m m})$ & Valor p \\
\cline { 2 - 5 } Parámetro & AL -L & $0,66 \pm 2,43$ & 0,26 & $0,92 \pm 2,56$ & 0,083 \\
Anterior & AL-NAI & $0,82 \pm 2,24$ & 0,13 & $1,13 \pm 2,6$ & $0,008^{*}$ \\
Parámetro & AL-L & $0,78 \pm 2,24$ & 0,073 & $0,13 \pm 2,08$ & 0,755 \\
Posterior & AL-NAI & $1,49 \pm 2,12$ & $<0,001^{* *}$ & $2,00 \pm 1,94$ & $<0,001^{* *}$ \\
\hline
\end{tabular}

* Valor $\mathrm{p}<0,01 ; * *$ Valor $\mathrm{p}<0,001$.

\section{DISCUSIÓN}

Establecer puntos de reparo anatómicos confiables relacionados con el NAI es un paso fundamental en la cirugía ortognática, donde la posición de estructuras como la língula mandibular es de vital importancia para realizar la SSRO. Sin embargo su posición es variable en diferentes poblaciones, por lo que necesario establecer estos parámetros para cada caso, y a veces es difícil de reconocer intraoperatoriamente debido a la escaza visión del campo quirúrgico debido a la presencia de uniones musculotendinosas y variantes morfológicas en la cara medial de la mandíbula, por lo que se ha atribuido una relevante importancia a la AL en la cara lateral de la rama mandibular para ser utilizada como guía quirúrgica tanto en la SSRO como en la osteotomía intraoral vertical de la rama mandibular (IVRO) (Aziz et al., 2007; Balcioglu et al., 2010). 
Al comparar la distancia longitudinal desde el parámetro superior (EM) hacia la AL y el NAI $(9,95 \pm 3,16 \mathrm{~mm}$ y 9,3 $\pm 3,39 \mathrm{~mm}$ lado derecho e izquierdo respectivamente), existe una mayor diferencia que desde la EM hacia AL y L $(4,23 \pm 2,97 \mathrm{~mm}$ y $3,63 \pm 3,14 \mathrm{~mm}$ lado derecho e izquierdo respectivamente), siendo estadísticamente significativa en ambos casos $(<0,001)$. Yu \& Wong al determinar la distancia vertical desde la EM a la AL obtuvieron una media de $14,8 \pm 2,90 \mathrm{~mm}$, y de EM a NAI de 21,6 $\pm 3,31 \mathrm{~mm}$, indicando que la distancia vertical media entre AL y el NAI fue de $6,7 \pm 1,68 \mathrm{~mm}$, con un máximo de $10,5 \mathrm{~mm}$ y un mínimo de $2,4 \mathrm{~mm}$; además los autores relatan que no existe diferencia estadística $(\mathrm{P}>0,05)$ cuando se comparan estos parámetros en relación al sexo, edad o lado de la mandíbula.

En las relaciones horizontales que mantiene la $\mathrm{AL}$ con el MA y MP, se encontró que la distancia entre el parámetro posterior tanto con AL como con NAI eran ampliamente mayores que AL comparada con L, siendo la primera medición estadísticamente significativa $(\mathrm{p}<0,01)$, mientras que esta diferencia no fue observada al tomar como referencia el parámetro anterior $(\mathrm{p}=0,13)$. Yu \& Wong al determinar la distancia horizontal desde AL y MA obtuvieron una media de $17,7 \pm 2,89 \mathrm{~mm}$ y la distancia de NAI al MA fue de 18,6 $\pm 2,49$, mientras que la diferencia media horizontal entre AL y NAI fue de 0,9 $\pm 2,42 \mathrm{~mm}$, siendo esta diferencia similar a nuestras mediciones, $0,82 \pm 2,24 \mathrm{~mm}$ en el lado derecho y 1,13 $\pm 2,6 \mathrm{~mm}$ en el lado izquierdo. Sin embargo los valores lineales entre los puntos anatómicos fueron menores en nuestra población (Tabla I y II). Recientemente Apinhasmit et al. investigaron la posición de la AL en relación con la $\mathrm{L}$ en mandíbulas secas tailandesas. La AL fue perceptible en el 80,4\% de los casos. La mayoría de las ALs se encontraron con una distancia máxima de $5,9 \mathrm{~mm}$ y $8,2 \mathrm{~mm}$ hacia anterior y superior. En un radio de $5 \mathrm{~mm}$ desde L se incluyen el 84,5\% de las ALs. Por lo tanto, un corte más de un $5 \mathrm{~mm}$ posterior o superior a la AL sería dentro de un área de estadística segura evitando lesionar el paquete neurovascular alveolar inferior que pasa inmediatamente a la L. Los autores recomiendan la utilización de la AL como punto anatómico quirúrgico, sobre todo en casos de IVRO donde no es posible la visualización directa del foramen mandibular o L. A diferencia de esta frecuencia de aparición, en nuestro estudio pudimos determinar la presencia de la AL en todos casos, similar a lo reportado por Pogrel et al. (1995) tras el exámen de 20 mandíbulas de cadáveres realizado por tres observadores independientes, se informó que la AL puede ser identificada en un $100 \%$, sin embargo, se reconoce en un radio de $5 \mathrm{~mm}$ a la L sólo en $43 \%$ de los casos y hubo concordancia exacta en sólo el 22,5\% de los casos.

Aziz et al. determinaron la fiabilidad de la utilización de la AL como una guía para la realización de la SSRO en 18 mandíbulas de cadáveres, encontrándose en todos los casos al igual que la L. Al analizar su posición, hubo concordancia completa de la posición de la L y AL en la dimensión antero-posterior en el 11,1\% de las muestras. En el 33\% de las muestras, la L se encontró anterior a la AL y en el $45,6 \%$ de la L se encontró posterior de la AL. Hubo concordancia completa de la L y AL en la dimensión superior-inferior en el 2,8\% de las muestras. La L fue encontrado superior a la AL en el 47,2\% de las muestras e inferior en el 50\% de las muestras. En la mayoría de los casos, la posición de la L fue postero-inferior con respecto a la posición de la AL. En una medida de $5 \mathrm{~mm}$ por detrás de la AL no había ningún riesgo de dañar el paquete neurovascular en este estudio cadavérico.

En nuestros resultados encontramos una diferencia significativa en sentido vertical entre la ubicación de AL y NAI, confirmando lo descrito por (Hogan \& Ellis), donde probablemente la prominencia ósea obedece a una respuesta ósea a los músculos y tendones que se insertan en esa zona en lugar de tener una relación morfológica con la entrada del NAI.

Los datos anatómicos medidos en este estudio para evaluar las relaciones geométricas entre AL, L y NAI mostraron una gran variedad confirmando las observaciones de Martone et al. Estas diferencias podrían estar dadas por la definición de ubicación del NAI. En el presente estudio, todos las ALs se encuentran superior a el punto más bajo de la entrada del NAI, al igual que lo reportado por Yu \& Wong. Según estos autores, si el ancho del paquete neurovascular se toma en cuenta, se puede suponer que algunas de las posiciones de AL estarían ubicadas a la misma altura o inferior al paquete neurovascular. En tales casos, la osteotomía debe realizarse encima de la posición a fin de evitar el estiramiento o compresión del nervio.

Las medidas de evaluación de las relaciones geométricas entre los puntos anatómicos mostraron que el uso de AL como guía quirúrgica puede ser confiable sólo en algunos casos. Si se utiliza, la osteotomía horizontal medial se debe hacer dentro de los $5 \mathrm{~mm}$ superior a la AL y extendido de 4-8 mm posterior a ella. Esta observación se puede inferir ya que la AL se ubica en su mayoría superior y anterior al NAI (Yu \& Wong), pero en nuestros resultados las relaciones geométricas de la AL y el NAI no siempre coinciden, pudiendo estar anterior o posterior, superior o inferior al NAI o incluso coincidir.

La posición geométrica de AL en relación con el NAI es de gran importancia clínica. Westermark et al. (1999) evaluaron las posibles correlaciones entre la disfunción neurosensorial y diversas variables en 496 cirugías de SSRO, 
sugiriendo que la disección de los tejidos blandos de la cara medial de la rama mandibular puede ser en parte responsable de la disfunción del NAI, por lo que un abordaje de la cara lateral y la utilización de sus reparos, como la AL es más segura. Jääskeläinen et al. (2000) monitorearon al NAI durante la cirugía de SSRO encontrando que los cambios más evidentes en la conducción nerviosa sensorial se producen durante la intervención de la cara medial de la rama mandibular al realizar el corte horizontal del hueso haciendo que se comprima y se estire por el uso de retractores. Con nuestros resultados, así como los reportados por otros autores (Yu \& Wong), se puede suponer que el NAI no podría estar en la posición esperada en relación con la AL.

En nuestros resultados $\mathrm{L}$ se ubicó en sentido vertical a $17,45 \pm 2,34 \mathrm{~mm}$ y $17,10 \pm 2,05 \mathrm{~mm}$ de la EM y a $34,01 \pm 3,96$ y $34,09 \pm 4,53$ de la EAG (lado derecho e izquierdo respectivamente). Kim et al. (1997) al estudiar la anatomia relacionada con la SSRO en 242 mandíbulas coreanas indican que la $\mathrm{L}$ se ubica a $15,1 \pm 2,3 \mathrm{~mm}$ de la EM en mandíbulas dentadas y a $14,5 \pm 3,5 \mathrm{~mm}$ en desdentadas, mientras que hacia la EAG se ubicó a $32,8 \pm 2,5 \mathrm{~mm}$ en mandíbulas dentadas y a $32,9 \pm 1,4 \mathrm{~mm}$ en desdentados. Fujimura et al. determinaron en mandíbulas secas y pacientes Japoneses la distancia de $\mathrm{L}$ a EM, reportando valores de $16,2 \pm 2,1 \mathrm{~mm}$ (rango 12-20) en mandíbulas secas y $15,6 \pm 2,6 \mathrm{~mm}$ (rango 12-21) en pacientes. Balcioglu et al. investigaron la posición de la L en cadáveres de Turquía, donde las distancias de L a EM fueron de $15,4 \pm 2,1 \mathrm{~mm}$ y de $\mathrm{L}$ a EAG $49,5 \pm 4,3 \mathrm{~mm}$ y no se encontraron diferencias significativas entre los lados derecho e izquierdo. Además, Kositbowornchai et al. (2007) en mandíbulas tailandesas describen una distancia de NAI a EM de $24,54 \pm 2,69$ y a EAG de $28,00 \pm 4,14$, similar a nuestros datos. Estos resultados indican que la posición de la L es más alta en nuestra población. Kositbowornchai et al. indican

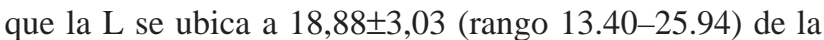
EM y a $35,79 \pm 3,38$ (rango $30,49-38,66$ ) de la EAG, demostrando su variabilidad posicional, lo que debe ser tomado en cuenta en la cirugía y al momento de realizar una adecuada técnica anestésica troncular mandibular.

En nuestra muestra el ancho promedio de la rama mandibular en sentido horizontal fue de $32,1 \pm 69$, lo que es concordante con los valores previos de $31,3 \pm 2,7 \mathrm{~mm}$ (Fujimura et al.), 31,03-32,7mm (da Fontura et al., 2002) y $35 \mathrm{~mm}$ (Kositbowornchai et al.). En relación a las estructuras ubicadas en este sentido, Fujimura et al. indican una distancia entre NAI y MA de $15,5 \pm 1,9 \mathrm{~mm}$ en mandíbulas secas y $17,1 \pm 2,6 \mathrm{~mm}$ en pacientes. Según estos autores el borde anterior del foramen mandibular se encuentra cerca del centro de la ancho de la rama mandibular (NAI-MA/NAI-MP) con una proporción de 0,49. En los pacientes, la proporción es de 0,53, ubicando al foramen mandibular ligeramente por detrás del centro de la ancho de la rama, en comparación con las mandíbulas secas. La punta de la L en ambos grupos (16,2 a 15,6 $\mathrm{mm}$ ) se encuentra cerca de un tercio de la distancia desde el centro de la EM hasta el borde inferior de la rama. Kim et al. reportan una distancia de $\mathrm{L}$ al MA de $17,5 \mathrm{~mm} \pm 2,6$ en mandíbulas dentadas y $17,3 \pm 2,4$ en desdentadas adultas, mientras que en relación al MP se ubicó a un $54 \%(15,2 \pm 2,8)$ del ancho de la rama mandibular en dentados y desdentados. Kositbowornchai et al. describen una distancia del NAI al MA de 20,70 $\pm 2,27 \mathrm{~mm}$ y al MP de $15,4 \pm 1,9 \mathrm{~mm}$, mientras que Malcioglu et al. presentan distancias de la L al MA de $18,1 \pm 2,7 \mathrm{~mm}$ y al MP $16,6 \pm 2,5 \mathrm{~mm}$. No se encontraron diferencias significativas entre los lados derecho e izquierdo, para todos los parámetros al igual que en nuestro reporte, donde observamos distancias similares, pero menores $(16,01 \pm 2,51 \mathrm{~mm}$ lado derecho y $16,19 \pm 2,21 \mathrm{~mm}$ lado izquierdo) entre L-MA y, mayores entre L-MP $(16,26 \pm 1,60 \mathrm{~mm}$ lado derecho y $15,46 \pm 1,77 \mathrm{~mm}$ lado izquierdo), mientras que el NAI mostró distancias al MA y MP de $16,16 \pm 1,69 \mathrm{~mm}$ $16,41 \pm 2,47 \mathrm{~mm}$ y, $13,99 \pm 1,40 \mathrm{~mm}-13,59 \pm 1,47 \mathrm{~mm}$ (derechaizquierda respectivamente). Esto indica que la posición en sentido horizontal de la L en nuestra muestra está ubicada más en el centro de la rama mandibular, coincidiendo con Fujimura et al., sin embargo el NAI muestra diferencias bilaterales, lo que podría estar causado por un desarrollo asimétrico en el lado izquierdo de la mandíbula. Según Kositbowornchai et al. la distancia MA-NAI fue mayor que la distancia MP-NAI, de tal manera que la L se situó ligeramente por detrás del centro del ancho de la rama mandibular.

El borde distal del 2MI mostró diferencias estadísticas entre el lado derecho e izquierdo en términos de las distancias a L, AL y NAI, determinando que las mandíbulas presentaron un crecimiento asimétrico, lo que fue confirmado por las mediciones angulares, posiblemente a nivel del cuerpo, ya que las distancias entre los parámetros ubicados dentro de la rama mandibular no muestran en general asimetría bilateral. Yu \& Wong reporta que la posición media del NAI con el 2MI no cambia significativamente entre los años 20-40 ni entre diferentes sexos bilateralmente. Nuestra distacia es levemente mayor en el lado derecho y significativamente mayor a lo reportada por Kim et al. $(28,7 \pm 2,87 \mathrm{~mm})$ en coreanos en el lado izquierdo.

Informes sugieren que la osteotomía horizontal media debería ser "justo por encima de la língula mandibular, y debe extenderse lo más lejos posible de la punta de la língula mandibular, con una longitud media de esta línea de $18 \mathrm{~mm}$ " (Dalpont; Epker). Yu \& Wong reportaron en población taiwanesa una distancia media horizontal del foramen mandibular a MA de $18,6 \pm 2,49 \mathrm{~mm}$, mayor a lo sugerido por los textos clásicos, mientras que según nuestros resultados es menor (16mm aproximadamente). Esto sugiere que 
la longitud de la línea debe ser de al menos $18 \mathrm{~mm}$ para algunos casos y hasta $20 \mathrm{~mm}$ en otros, dependiendo de las características anatómicas de cada población, mientras que en sentido vertical se recomienda que el corte se efectue al menos sobre 4-5 mm superior y posterior de la L para prevenir el daño del NAI (Muto et al.; Kositbowornchai et al.).

Junto a una adecuada determinación de guías para la cirugía, es importante tener en cuenta las estructuras anatómicas que podrían causar complicaciones como hemorragia, donde existen muchos vasos en estrecha proximidad a la zona de osteotomía, las que incluyen la arteria carótida interna y externa, la vena retromandibular, la vena facial y vasos relacionados con el NAI. Las complicaciones en estos vasos pueden requerir transfusión de sangre o la reintervención (Teltzrow et al., 2005). Se ha determinado la distancia existente entre la EM y la arteria maxilar ubicán-

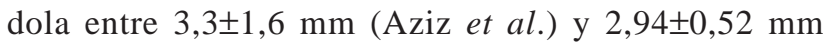
(Balcioglu et al.). En relación al daño nervioso, Teltzrow et al. relatan la sección del NAI en 27 pacientes, representando solo el daño visto durante la operación, pero el índice de trauma del nervio podría ser mayor. Esto concuerda con lo reportado por Al-Bishri et al. (2005), para quienes existe mayor daño neurosensorial en la cirugía de SSRO $(11,6 \%)$ en comparación con la osteotomías vertical Intraoral de la rama (7,5\%), mientras que Kallal et al. (2007) relatan el desarrollo de otras alteraciones como un neuroma traumático en el NAI posterior a la SSRO.

Las referencias anatómicas y las estructuras de riesgo deben ser tomadas en cuenta sobre todo en las recientes modificaciones de la técnica quirúrgica Obwegeser-Dal Pont, donde se debe realizar una osteotomía adicional en el borde inferior de la mandíbula (Böckmann et al., 2011; Schoen et al., 2011).
Existen muchas técnicas que se pueden utilizar en la realización de la SSRO, pero ninguna técnica específica puede ser apropiada para cada situación. El cirujano debe elegir un plan de tratamiento adecuado y realizar un procedimiento quirúrgico correcto, teniendo en cuenta varios factores que incluyen la posición anatómica de L, AL, NAI y foramen mandibular (Cillo \& Stella, 2005). Según Kositbowornchai et al. las mediciones utilizando estructuras como L o FM en las radiografías panorámicas no presenta valores reales en comparación con las mediciones mandibulares directas como se realizó en este estudio debido al efecto de la angulación radiográfica. Sin embargo, las observaciones radiografica de estas estructuras son necesarias para una correcta planificación y guía, teniendo en cuenta valores basales en cada población como los provistos por nuestra investigación. En la práctica clínica recomendamos confirmar estos parámetros mediante las imágenes de TC antes de la operación.

En conclusión, no existen diferencias al realizar una medición para fines quirúrgicos en la rama izquierda o derecha, es decir, si al paciente le falta alguna sección de la mandíbula o no es clara en radiografías, se podría utilizar como referencia la otra rama mandibular. La AL se relaciona más con la L que con el NAI si se miden desde el parámetro anterior y posterior. No recomendamos el uso de la $\mathrm{AL}$ como único punto de referencia anatómico, ya que presenta diferencias en relación a L y NAI, pero se aconseja realizar los procedimientos $5 \mathrm{~mm}$ sobre y posterior a ella. En el caso de que sólo se tenga la AL como referencia para encontrar la L, es más seguro medirla desde la EAG o desde los bordes de la rama mandibular manteniendo distancias de seguridad para disminuir el riesgo de lesión nerviosa al realizar una SSRO.

SALGADO, G.; INZUNZA, O.; CANTÍN, M.; FUENTES, F.; INOSTROZA, V.; ERRÁZURIZ, M. J. \& PAVEZ, C. Evaluation of mandibular anatomy related to sagittal split ramus osteotomy. Int. J. Morphol., 30(1):30-39, 2012.

SUMMARY: Sagittal split ramus osteotomy (SSRO) of the mandible is one of the most common surgical techniques to correct mandibular deformities. In order to prevent many surgical anatomical problems, surgeons have found that further investigation of the anatomical structures related to SSRO is needed. This study aims to investigate positions of the antilingula (AL), inferior alveolar nerve (IAN) and other anatomic landmarks in relation to the lingula of dried mandibles. 70 Chilean dried hemimandibles were studied. The AL, others landmarks and the corresponding position of the $\mathrm{L}$ were marked on the internal and external aspect of the mandibular ramus respectively. The distances from the AL, IAN and L were measured in the anterior-posterior and the superior-inferior planes using a digital caliper and geometric relationship was established. The results showed the AL was discernible in $100 \%$ of lateral mandibular rami studied. The most of the AL was found anteriorly to the $\mathrm{L}$ with a mean distance of $0.66 \pm 2.43 \mathrm{~mm}$ and $0.92 \pm 2.56 \mathrm{~mm}$, and $4.23 \pm 2.97 \mathrm{~mm}$ and $3.62 \pm 3.14 \mathrm{~mm}$ superiorly (right-left sides respectively) (both with p value $<0.001$ ). Similar values were seen in relation with IAN. No significant differences were found between the right and left sides, for the majority of parameters. The studied parameters will assist clinicians to determine the anatomical proximity to the IAN, and, minimize the risk of damaging nerver and vessels. We do not recommend the use of antilingula as only anatomical landmark when performing a SSRO procedure. nerve.

KEY WORDS: Sagittal split ramus osteotomy; Mandible; Anatomical landmarks; Antilingula; Lingula; inferior alveolar 


\section{REFERENCIAS BIBLIOGRÁFICAS}

Al-Bishri, A.; Barghash, Z.; Rosenquist, J. \& Sunzel, B. Neurosensory disturbance after sagittal split and intraoral vertical ramus osteotomy: as reported in questionnaires and patients' records. Int. J. Oral Maxillofac. Surg., 34(3):247$51,2005$.

Apinhasmit, W.; Chompoopong, S.; Jansisyanont, P.; Supachutikul, K.; Rattanathamsakul, N.; Ruangves, S. \& Sangvichien, S. The study of position of antilingula, midwaist of mandibular ramus and midpoint between coronoid process and gonion in relation to lingula of 92 Thai dried mandibles as potential surgical landmarks for vertical ramus osteotomy. Surg. Radiol. Anat., 33(4):337-43, 2011.

Aziz, S. R.; Dorfman, B. J.; Ziccardi, V. B. \& Janal, M. Accuracy of using the antilingula as a sole determinant of vertical ramus osteotomy position. J. Oral Maxillofac. Surg., 65(5):859-62, 2007.

Balcioglu, H. A.; Kilic, C.; Varol, A.; Ozan, H.; Kocabiyik, N. \& Yildirim, M. A Morphometric Study of the Maxillary Artery and Lingula in Relation to Mandibular Ramus Osteotomies and TMJ Surgery. Eur. J. Dent., 4(2):166-70, 2010.

Böckmann, R.; Schön, P.; Frotscher, M.; Eggeler, G.; Lethaus, B. \& Wolff, KD. Pilot study of modification of the bilateral sagittal split osteotomy (BSSO) in pig mandibles. $J$. Craniomaxillofac. Surg., 39(3):169-72, 2011.

Cillo, J. E. \& Stella, J. P. Selection of sagittal split ramus osteotomy technique based on skeletal anatomy and planned distal segment movement: current therapy. J. Oral Maxillofac. Surg., 63(1):109-14. 2005.

da Fontoura, R. A.; Vasconcellos, H. A. \& Campos, A. E. Morphologic basis for the intraoral vertical ramus osteotomy: anatomic and radiographic localization of the mandibular foramen. J. Oral Maxillofac. Surg., 60(6):660-6, 2002.

Dal Pont, G. Retromolar osteotomy for the correction of prognathism. J. Oral Surg., Anesth. \& Hosp. D. Serv., 19:427, 1961.

Epker, B. N. Modifications in the sagittal osteotomy of the mandible. J Oral Surg., 35(2):157-9, 1977.

Fridrich, K. L.; Holton, T. J.; Pansegrau, K. J. \& Buckley, M. J. Neurosensory recovery following the mandibular bilateral sagittal split osteotomy. J. Oral Maxillofac. Surg., 53(11):1300-7, 1995.

Fujimura, K.; Segami, N. \& Kobayashi, S. Anatomical study of the complications of intraoral vertico-sagittal ramus osteotomy. J. Oral Maxillofac. Surg., 64(3):384-9, 2006.
Guernsey, L. H. \& DeChamplain, R. W. Sequelae and complications of the intraoral sagittal osteotomy in the mandibular rami. Oral Surg. Oral Med. Oral Pathol., 32(2):176-92, 1971.

Hayward, J.; Richardson, E. R. \& Malhotra, S. K. The mandibular foramen: its anteroposterior position. Oral Surg. Oral Med. Oral Pathol., 44(6):837-43, 1977.

Hogan, G. \& Ellis, E. 3rd. The "antilingula"--fact or fiction? J. Oral Maxillofac. Surg., 64(8):1248-54, 2006.

Hunsuck, E. E. A modified intraoral sagittal splitting technique for correction of mandibular prognathism. J. Oral Surg., 26(4):250-3, 1968.

Jääskeläinen, S. K.; Teerijoki-Oksa, T.; Forssell, K.; Vähätalo, K.; Peltola, J. K. \& Forssell, H. Intraoperative monitoring of the inferior alveolar nerve during mandibular sagittalsplit osteotomy. Muscle Nerve, 23(3):368-75, 2000.

Kallal, R. H.; Ritto, F. G.; Almeida, L. E.; Crofton, D. J. \& Thomas, G. P. Traumatic neuroma following sagittal split osteotomy of the mandible. Int. J. Oral Maxillofac. Surg., 36(5):453-4, 2007.

Kim, H. J.; Lee, H. Y.; Chung, I. H.; Cha, I. H. \& Yi, C. K. Mandibular anatomy related to sagittal split ramus osteotomy in Koreans. Yonsei Med. J., 38(1):19-25, 1997.

Klinge, B.; Petersson, A. \& Maly, P. Location of the mandibular canal: comparison of macroscopic findings, conventional radiography, and computed tomography. Int. J. Oral Maxillofac. Implants, 4(4):327-32, 1989.

Kositbowornchai, S.; Siritapetawee, M.; Damrongrungruang, T.; Khongkankong, W.; Chatrchaiwiwatana, S.; Khamanarong, K. \& Chanthaooplee, T. Shape of the lingula and its localization by panoramic radiograph versus dry mandibular measurement. Surg. Radiol. Anat., 29(8):689-94, 2007.

Martone, C. H.; Ben-Josef, A. M.; Wolf, S. M. \& Mintz, S. M. Dimorphic study of surgical anatomic landmarks of the lateral ramus of the mandible. Oral Surg. Oral Med. Oral Pathol., 75(4):436-8, 1993.

Muto, T.; Shigeo, K.; Yamamoto, K. \& Kawakami, J. Computed tomography morphology of the mandibular ramus in prognathism: effect on the medial osteotomy of the sagittal split ramus osteotomy. J. Oral Maxillofac. Surg., 61(1):8993, 2003.

O'Ryan, F. Complications of orthognathic surgery. Oral Maxillofac. Surg. Clin. North. Am., 2:593-613, 1990. 
Plooij, J. M.; Naphausen, M. T.; Maal, T. J.; Xi, T.; Rangel, F. A.; Swennnen, G.; de Koning, M.; Borstlap, W. A. \& Bergé, S. J. 3D evaluation of the lingual fracture line after a bilateral sagittal split osteotomy of the mandible. Int. J. Oral Maxillofac. Surg., 38(12):1244-9, 2009.

Pogrel, M. A.; Schmidt, B. L. \& Ammar, A. The presence of the antilingula and its relationship to the true lingula. $\mathrm{Br}$. J. Oral Maxillofac. Surg., 33(4):235-8, 1995.

Precious, D. S.; Lung, K. E.; Pynn, B. R. \& Goodday, R. H. Presence of impacted teeth as a determining factor of unfavorable splits in 1256 sagittal-split osteotomies. Oral Surg. Oral Med. Oral Pathol. Oral Radiol. Endod., 85(4):362-5, 1998.

Rajchel, J.; Ellis, E. 3rd. \& Fonseca, R. J. The anatomical location of the mandibular canal: its relationship to the sagittal ramus osteotomy. Int. J. Adult Orthodon. Orthognath. Surg., 1(1):3747, 1986

Ribeiro, D. P.; Gandelmann, I. H. \& Medeiros, P. J. Comparison of mandibular rami width in patients with prognathism and retrognathia. J. Oral Maxillofac. Surg., 64(10):1506-9, 2006.

Schoen, P.; Frotscher, M.; Eggeler, G.; Kessler, P.; Wolff, K. D. \& Boeckmann, R. Modification of the bilateral sagittal split osteotomy (BSSO) in a study using pig mandibles. Int. J. Oral Maxillofac. Surg., 40(5):516-20, 2011.

Teltzrow, T.; Kramer, F. J.; Schulze, A.; Baethge, C. \& Brachvogel, P. Perioperative complications following sagittal split osteotomy of the mandible. J. Craniomaxillofac. Surg., 33(5):307-13, 2005.

Torres, J. H.; Nomdedeu, H. \& Leboucq, N. Localization of the mandibular canal: experimental comparison of four types of radiological examination. Actual Odontostomatol., 45(174):191-8, 1991.

Trauner, R. \& Obwegeser, H. The surgical correction of mandibular prognathism and retrognathia with consideration of genioplasty. I. Surgical procedures to correct mandibular prognathism and reshaping of the chin. Oral Surg. Oral Med. Oral Pathol., 10(7):677-89, 1957.

Turvey, T. A. Intraoperative complications of the sagittal split osteotomy of the mandibular ramus; incidence and management. J. Oral Maxillofac. Surg., 43:504-9, 1985.

Van Sickels, J. E.; Jeter, T. S. \& Theriot, B. A. Management of an unfavorable lingual fracture during a sagittal split osteotomy. J. Oral Maxillofac. Surg., 43(10):808-9, 1985.

Westermark, A.; Englesson, L. \& Bongenhielm, U. Neurosensory function after sagittal split osteotomy of the mandible: a comparison between subjective evaluation and objective assessment. Int. J. Adult. Orthodon. Orthognath. Surg., 14(4):268-75, 1999.
Yates, C.; Olson, D. \& Guralnick, W. The antilingula as an anatomic landmark in oral surgery. Oral Surg. Oral Med. Oral Pathol., 41(6):705-8, 1976.

Yu, I. H. \& Wong, Y. K. Evaluation of mandibular anatomy related to sagittal split ramus osteotomy using 3-dimensional computed tomography scan images. Int. J. Oral Maxillofac. Surg., 37(6):521-8, 2008.

\section{Dirección para correspondencia: \\ Dr. Guillermo Salgado Alarcón \\ Facultad de Medicina \\ Pontificia Universidad Católica de Chile \\ Santiago \\ CHILE}

Email: gsalgado@med.puc.cl

Recibido : 14-10-2011

Aceptado: 21-11-2011 